\title{
BMJ Open Experiences of community-dwelling older adults living with multiple chronic conditions: a qualitative study
}

\author{
Jenny Ploeg, ${ }^{01}$ Marta Canesi, ${ }^{2}$ Kimberly D Fraser, ${ }^{3}$ Carrie McAiney, ${ }^{4}$ \\ Sharon Kaasalainen, ${ }^{5}$ Maureen Markle-Reid, ${ }^{1}$ Sinead Dufour, ${ }^{6}$ Lisa Garland Baird, ${ }^{3}$ \\ Tracey Chambers ${ }^{1}$
}

To cite: Ploeg J, Canesi M, D Fraser $\mathrm{K}$, et al. Experiences of community-dwelling older adults living with multiple chronic conditions: a qualitative study. BMJ Open 2019:9:e023345. doi:10.1136/ bmjopen-2018-023345

- Prepublication history for this paper is available online. To view these files, please visit the journal online (http://dx.doi org/10.1136/bmjopen-2018023345).

Received 3 April 2018

Revised 21 January 2019

Accepted 13 February 2019

Check for updates

(c) Author(s) (or their employer(s)) 2019. Re-use permitted under CC BY-NC. No commercial re-use. See rights and permissions. Published by BMJ.

${ }^{1}$ School of Nursing, Aging, Community and Health Research Unit, McMaster University, Hamilton, Ontario, Canada

${ }^{2}$ School of Nursing, Universita degli Studi di Milano-Bicocca, Milano, Lombardia, Italy

${ }^{3}$ Faculty of Nursing, University of Alberta, Edmonton, Alberta, Canada

${ }^{4}$ Department of Psychiatry and Behavioural Neurosciences,

McMaster University, Hamilton,

Ontario, Canada

${ }^{5}$ School of Nursing, McMaster University, Hamilton, Ontario,

Canada

${ }^{6}$ School of Rehabilitation Science, McMaster University, Hamilton, Ontario, Canada

Correspondence to

Dr Jenny Ploeg;

ploegj@mcmaster.ca

\section{ABSTRACT}

Objectives The aim of the study was to understand the experiences of living with multiple chronic conditions (MCC) from the perspective of community-living older adults with MCC.

Design A qualitative study using an interpretive description approach.

Setting Participants were recruited from southern Ontario, Canada.

Participants 21 community-living, older adults ( $\geq 65$ years) with an average of 7.4 chronic conditions including one of diabetes, dementia or stroke.

Methods Data were collected through digitally-recorded, in-depth, semi-structured in-person interviews. Interview transcripts were analysed and coded using Thorne's interpretive description approach.

Results Five themes were identified representing older adults' experiences of living with MCC: (a) trying to stay healthy while living with MCC, (b) depending on family caregivers for support with just about everything, (c) paying the high costs of living with MCC, (d) making healthcare decisions by proxy and (e) receiving healthcare services that do not address the complex needs of persons living with MCC.

Conclusions The experience of living with MCC in the community was complex and multi-faceted. The need for a person-centred and family-centred approach to care in the community, which includes the coordination of health and social services that are tailored to the needs of older adults and their informal caregivers, was underscored. Such an approach would facilitate improved informationsharing and discussion of care management options between health professionals and their patients, enable older adults with MCC to actively engage in priority-setting and decision-making and may result in improved health and quality of life for older adults with MCC.

\section{INTRODUCTION}

The presence of 'multiple chronic conditions' (MCC), for any given person, is defined when they have been diagnosed with two or more concurrent chronic conditions, such as diabetes and dementia. ${ }^{2}$ This is a common experience, ${ }^{3}$ with increasing prevalence with age ${ }^{4}$ and for women. ${ }^{56}$ Predictably, the

\section{Strengths and limitations of this study}

- Study findings represent the views of vulnerable older adults who have on average 7.4 chronic conditions.

- Participants were recruited from multiple settings (eg, primary care, community support groups, media) and reflected variation in demographic characteristics of age, education, income and number and type of chronic conditions.

- The findings provide practical recommendations for improving care for older adults with MCC that can be used in the training of healthcare providers or as part of a complex intervention study.

- All study participants were Caucasian and Englishspeaking, therefore future research with a more culturally diverse sample would be important to understand unique perspectives and differences among older adults with MCC.

presence of MCC increases the risk for an individual in terms of mortality, impaired quality of life, disability, increased frequency of interactions with the healthcare system as well as the negative impact of polypharmacy. ${ }^{5-7}$

Qualitative research on the experience of older adults living with MCC has increased in the past 5 years. ${ }^{8-10}$ However, most qualitative studies have explored very specific issues such as symptom burden, ${ }^{11}$ gendered experiences, ${ }^{12}$ care practices, ${ }^{13}$ coping, ${ }^{14}$ health-related decision-making ${ }^{15}$ and desired care processes, ${ }^{16}$ rather than the overall experience of living with MCC. Furthermore, study participants were often drawn from single organisations that were not broadly representative of the diversity of community settings. ${ }^{17} 18$ Therefore, research is needed on the overall experience of living with MCC from the perspective of a broader group of community-living older adults to inform the design of approaches to MCG that could improve outcomes for this vulnerable group. 
The research question for this study was: What are the experiences of living with MCC from the perspective of older adults residing in the community? This paper, focusing specifically on older adults with MCC, used a subset of data from a larger Canadian study that explored the experiences of managing MCC among older adults with MCC, informal caregivers and healthcare providers (HCP) ${ }^{19}$

\section{METHODS}

\section{Study design}

We used Thorne's qualitative methodology, interpretive description (ID),${ }^{20}$ that addresses clinical questions using a flexible structure for inductively describing a phenomenon and understanding it from the perspective of those experiencing it. ${ }^{21}$ ID acknowledges 'the constructed and contextual nature of human experience that at the same time allows for shared realities'. (Thorne et al, p3) ${ }^{21}$ Two philosophical underpinnings of the ID design and this research are that: (a) reality is subjective, constructed, complex and contextual; and (b) the researcher and researched interact to produce research understandings. $^{21}$ ID studies are focused on clinical realities, conducted in naturalistic contexts and are intended to positively impact clinical care..$^{2021}$

\section{Study setting and sample}

Purposeful sampling strategies were used to recruit older adults with MCC including criterion sampling and maximum variation sampling. Eligible participants: (a) were $\geq 65$ years, (b) were community-dwelling, (c) were English-speaking, (d) had three or more chronic conditions and (e) had at least one chronic condition that included diabetes, dementia or stroke. These conditions were selected because they are among the leading causes of death among Canadians and are associated with high use of healthcare services. ${ }^{22}{ }^{23}$ Maximum variation sampling ensured that there was diversity among older adults in relation to age, gender and chronic conditions; we monitored the demographic characteristics of participants as they were recruited, and tried to ensure approximately equal proportions of older adults who: (a) were male and female; (b) were 67-74 years and 75 and over; and (c) had each of the three chronic conditions as above. These sampling decisions were informed by the literature we reviewed on MCC. Recruitment and analysis were conducted in parallel. Data collection ended when we had some confidence that the variation and complexity of participant responses were addressing the research question, recognising that there is always more to study on the topic. ${ }^{20}$

Eligible participants were identified from: (a) the patient rosters of two primary care practice settings in southern Ontario, Canada; (b) persons receiving support from the local Alzheimer Society and (c) through media postings (local newspapers, senior centres and on partner websites). In the ongoing analysis, we identified the

\section{Box 1 Sample interview questions}

- Tell me about your experiences in living with more than one chronic condition at a time.

- What do you do to prevent your chronic conditions from getting worse or new ones from developing?

- How do you make decisions about what chronic conditions or symptoms to manage first?

What helps you to manage your chronic conditions?

- What are some challenges you face in living with more than one chronic condition?

- What supports do you receive to help you live with more than one condition at a time?

- Older adults living with more than one condition often have multiple healthcare providers and services. How is your care coordinated among these providers and services?

- Have you talked with health professionals about managing more than one chronic condition at a time? Tell me about that.

- How have you worked with health professionals in making decisions about your care?

- What do you hope to achieve in your management of more than one chronic condition at a time?

need to better understand perspectives of persons with dementia, so the final three participants were recruited from the local Alzheimer Society. The main recruitment sites received an honorarium of $\$ 3000$ (Canadian dollars). All participants received a $\$ 25$ honorarium. Community-based programme representatives, not caring directly for the older adult, identified and contacted potential participants by phone. At the time of the first phone call to participants, the Research Coordinator asked each person if they had been diagnosed with any of a list of 20 chronic conditions. Only those with three or more conditions were included in the study. Research Coordinators provided additional information for participants, confirmed their study eligibility and arranged a mutual location and time for the interview.

\section{Data collection}

Data were collected from July 2013 to November 2013 through individual face-to-face digitally recorded semistructured interviews of $1-1.5$ hours using an interview guide informed by relevant literature. Box 1 provides a sample of the interview questions. The interview guide consisted of 20 questions with a number of probes for each question. Based on a pilot interview, we simplified the wording of some questions and added probes. Participants were encouraged to introduce novel ideas throughout the interview through the use of: (a) a broad opening question related to their experiences of living with MCC, (b) probes such as 'is there anything else' throughout the interview and (c) a final question asking if there was anything else they wanted to share. Experienced research coordinators, trained in consent and data collection procedures, interviewed participants. For individuals who had cognitive issues related to stroke or dementia, the Research Coordinator ensured simple language was used and extra time was provided in 


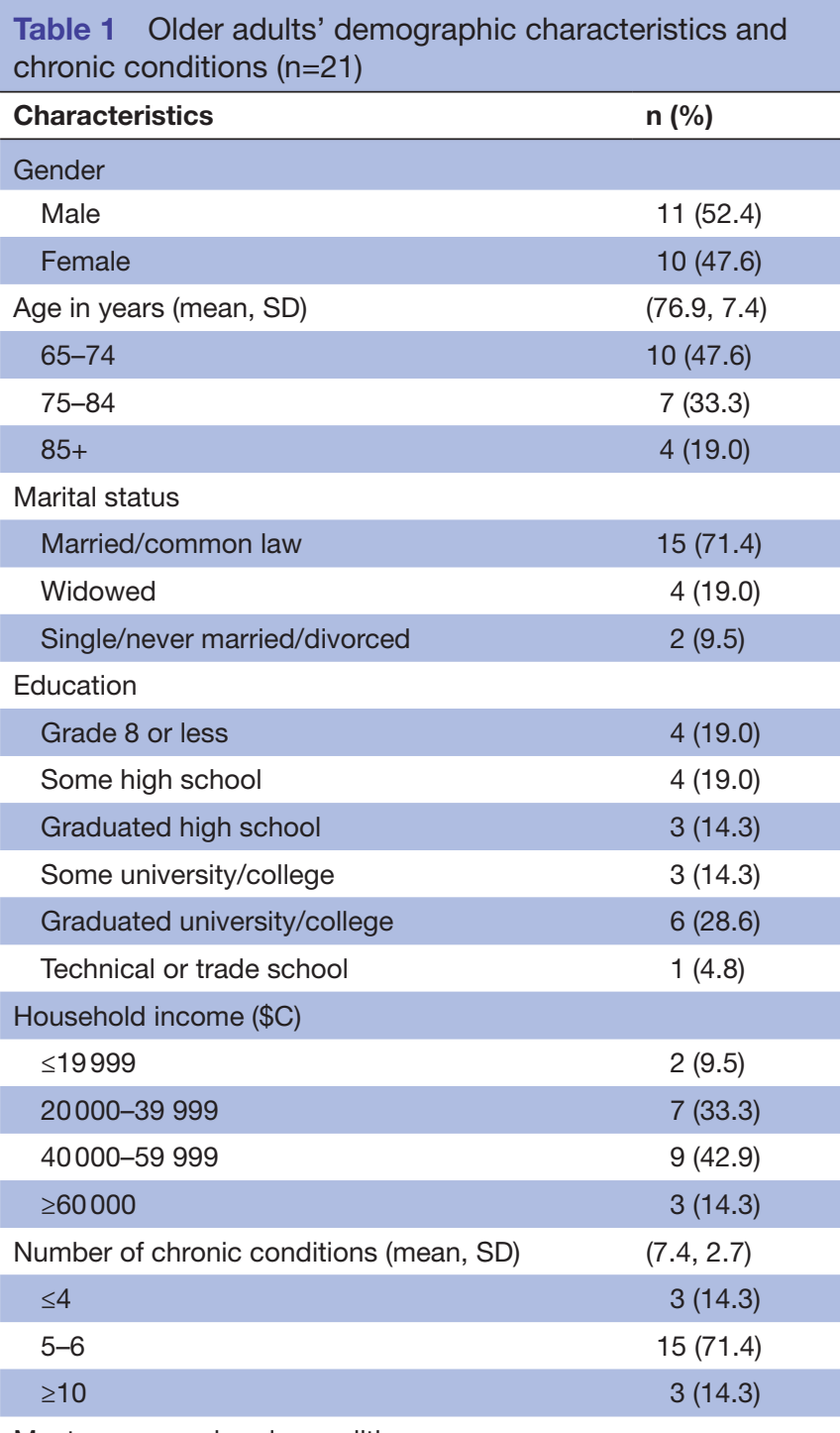

Most common chronic conditions

\begin{tabular}{lc}
$\begin{array}{l}\text { Cardiovascular disease (ischaemic heart } \\
\text { disease, arrhythmia) }\end{array}$ & $18(85.7)$ \\
Hypertension & $17(80.9)$ \\
Diabetes $^{*}$ & $15(71.4)$ \\
\hline $\begin{array}{l}\text { Arthritis or rheumatoid arthritis } \\
\text { Dementia and other memory conditions } \\
\text { (Alzheimer's disease, mild cognitive impairment, } \\
\text { vascular cerebral atrophy) }\end{array}$ & $11(61.9)$ \\
$\begin{array}{l}\text { Stroke and transient ischaemic attack* } \\
\text { Depression or anxiety }\end{array}$ & $11(52.4)$ \\
Asthma, chronic obstructive pulmonary disease & $6(28.6)$ \\
or chronic bronchitis & $10(47.6)$ \\
Vision disorders & $6(28.6)$ \\
Recruitment source: & \\
Family practice setting 1 & $9(42.9)$ \\
Family practice setting 2 & $6(28.6)$ \\
Alzheimer Society & $3(14.3)$ \\
Media postings & $3(14.3)$
\end{tabular}

*Participants had to have one of these three conditions to be eligible. conducting interviews. In cases where a spouse or family caregiver was present in the home at the time of the interview, the Research Coordinator asked them to kindly leave the room while the interview was taking place, so that the interview could focus on understanding the perspective of the participant.

\section{Data analysis}

Digital recordings of interviews were transcribed verbatim by a trained transcriptionist and cleaned for accuracy by a research assistant. We used inductive thematic analysis $^{24}$ as an analytic approach, which is consistent with the ID design ${ }^{25}$ and has been used in other ID studies. Our theoretical positioning was consistent with the two philosophical underpinnings of ID described previously. We followed the 6 steps of thematic analysis. In step 1, becoming familiar with the data, three research team members (two qualitative experts and one graduate student) each read through all transcripts and noted preliminary ideas about possible themes. We also reflexively documented our own feelings and reactions to the transcripts and how these influenced our understandings of the data, and discussed these in our team meetings. In step 2, performing coding, the three team members developed a coding scheme inductively from the data based on independent review of five transcripts. We met to reach agreement on a final coding scheme and two people used this to code all transcripts using NVivo V.11.0 to assist with data management. In steps 3 and 4, seeking themes and reviewing themes, the three team members met weekly over 3 months to identify recurring and converging themes. We used constant comparative analysis to identify similarities and differences in themes across participants. The entire research team then reviewed the themes and data within each theme and made suggestions for the final themes. In step 5, we created definitions of themes and named each theme. In step 6 , we developed a written report of the themes generated.

\section{Methodological integrity and rigour}

Credibility, or the accurate reflection of the experiences of participants ${ }^{26}$ was achieved through transparent descriptions of the research process and investigator triangulation that involved analysis conducted by investigators with divergent backgrounds. Transferability, or the ability to apply findings to similar contexts, was met through clear descriptions of the participants, settings and the research process. ${ }^{20}{ }^{26}$ Maximum variation sampling facilitated diverse perspectives and enhanced the transferability of findings. ${ }^{27}$ Dependability, or the consistency and quality of the inquiry processes over time, ${ }^{26}$ was addressed though the use of field notes and an audit trail of study decisions. Confirmability, the degree to which the data and interpretations are grounded in real events, ${ }^{26}$ was met by including participants' direct quotes in the findings and staying true to their exact words. Direct quotes in this paper have been labelled with participant numbers. 
Table 2 Participants' description of support received from family members

\begin{tabular}{lc|}
\hline Characteristics & $\mathbf{n}(\%)$ \\
\hline $\begin{array}{l}\text { Family member provides support with managing } \\
\text { MCC }\end{array}$ \\
\hline Yes & $19(90.5)$ \\
No & $2(9.5)$
\end{tabular}

Family member's relationship to older adult with $\operatorname{MCC}(n=19)^{*}$

$\begin{array}{lr}\text { Spouse } & 15(78.9) \\ \text { Daughter or son } & 12(63.2) \\ \text { Sibling } & 3(15.8) \\ \text { Other } & 2(10.5)\end{array}$

Types of care provided to older adults by family members $(n=19)$

A. Management of care

Discuss care of older adult with health professionals

$13(68.4)$

Manage finances

$11(57.9)$

Make decisions about care of older adult

$9(47.4)$

Make appointments

9 (47.4)

Complete forms

8 (42.1)

B. Daily activities

Getting around the home

$14(73.7)$

Shopping

$14(73.7)$

Cleaning

$13(68.4)$

Cooking

$11(57.9)$

Laundry

$11(57.9)$

Medication management

$10(52.6)$

Home maintenance

8 (42.1)

C. Personal care

Dressing 5 (26.3)

Bathing and toileting

$3(15.8)$

Help with medical equipment

$3(15.8)$

D. Social and emotional support

Conversations

$18(94.7)$

Companionship

$17(89.5)$

Comfort

$16(84.2)$

*Multiple responses are possible.

MCC, multiple chronic conditions.

\section{Patient involvement}

This study was conducted through the Aging, Community and Health Research Unit (ACHRU) at McMaster University (http://achru.mcmaster.ca). Researchers within ACHRU work with a Stakeholder Advisory Group (including patients and caregivers) to identify research priorities, conduct studies and disseminate findings with the goal of promoting optimal ageing at home. All participants received a mailed summary of the study findings.

\section{Ethical considerations}

This study was conducted in accordance with the Tri-Council Policy Statement, Ethical Conduct for Research Involving Humans. ${ }^{28}$ Written informed consent was obtained from all participants prior to data collection. Older adults who had dementia were deemed capable to consent if they met the following three criteria identified by Cohen-Mansfield ${ }^{29}$ : (1) understood the proposed research activities, (2) made an informed decision concerning willingness to participate in those activities and (3) communicated this decision to the Research Coordinator.

\section{RESULTS}

Demographic characteristics, chronic conditions and support Twenty-one older adults (mean age $=76.9$ years, $\mathrm{SD}=7.4$ years) participated in the study (table 1 ). There were similar proportions of male $(52.4 \%)$ and female $(47.6 \%)$ participants. Older adults reported having between 3 and 13 chronic conditions (mean=7.4, SD=2.7). All participants were Caucasian. Participants were recruited from two primary care practice settings $(n=15)$, the local Alzheimer Society $(n=3)$ and media postings $(n=3)$. Most older adults $(90.5 \%)$ reported having a family member as a caregiver (table 2). These caregivers provided a wide range of support including help with daily activities, personal care, managing care and social and emotional support.

\section{Experiences of living with MCC}

Older adults' experiences of living with MCC were characterised by five themes: (1) trying to stay healthy while living with MCC, (2) depending on family caregivers for support with just about everything, (3) paying the high costs of living with MCC, (4) making healthcare decisions by proxy and (5) receiving healthcare services that do not address the complex needs of persons living with MCC.

\section{Trying to stay healthy while living with MCC}

Older adult participants tried to stay as healthy as they could while recognising that they were living with MCC that were progressively worsening over time. They explained that "you just do your best at looking after yourself.' (P07) Most participants talked about engaging in physical activity and eating healthy foods to promote health and prevent their chronic conditions from getting worse. Walking was one of the most commonly mentioned activities; 'Well, I just keep on top of everything and make sure that I move...I walk a lot.' (P07) Some participants felt that physical activity was limited by chronic conditions and they paced themselves given their declining health: 'I try to go for walks. I have to go slowly. Before, I could walk as much as I wanted.' (P17)

Individuals living with diabetes spoke extensively about eating well as a way to manage MCC: 'I haven't touched dessert with any amount of sugar in it. I basically stick to the diet...And my blood sugar has been staying down.' 
(P06) One individual made major changes to his smoking and alcohol use after a new diagnosis of a chronic heart condition: 'That's another thing to deal with, the addictions. I went to a treatment centre and today I don't even think about a drink... and then the smoking...it's been a year and 5 days [without].' (P09)

Participants described maintaining mental and emotional health through positive attitudes and coping strategies. Most older adults held a positive attitude to life in spite of living with MCC: 'My life doesn't revolve around illness at all' (P01); 'I don't look on it as managing more than one condition at a time; it's just a matter of managing my life and getting the best out of my life and putting as much into it as I can.' (P13) Participants learnt to accept the reality of MCC in order to cope with new and ongoing conditions: 'I've adapted to it as it's happened; you get this problem and then you get this problem and then you have another problem and I've just dealt with it.' (P11)

Participants described staying healthy while living with MCC through active engagement in social and community activities: 'I do mostly volunteer work; get out and do things...I have a circle of friends who meet once a month.' (P01) These activities gave participants a sense of purpose and connection. Some individuals who were more limited in their activities due to their MCC made connections through social media: 'I get a lot of support from friends and the people I know on Facebook.' (P07) Finally, individuals spoke of the importance of being part of a spiritual community: 'I belong to three groups of my church... and an Alzheimer's group...they offer friendship, and we laugh.' (P21)

Participants living with MCC were prescribed multiple medications and they explained that taking these medications as prescribed was a way to stay healthy: 'What do you do to stay healthy? Just take pills.' (P20) They talked about being careful with their medications; one person described it as a form of vigilance: 'I am vigilant about taking the medication that's prescribed for me.' (P10) One of the main challenges participants experienced, sometimes related to cognitive issues, was forgetting to take their medications: 'I forgot one night to take them and I went over to the hospital... they told me that I should get them done up in a dosette and they're all labelled for the day.' (P06) Many participants described the use of such clearly labelled weekly packages of medications in order to manage their complex medication regimens.

Participants described keeping lists of medications and sharing these with doctors and pharmacists. They often worked with pharmacists to manage their medications; some talked about having an annual medication review to 'go over the medications and identify any changes and confirm what the current level of dosage is.' (P10) Participants also obtained information from pharmacists about possible drug interactions when new medications were added to an already long list of medications: 'I have often called the druggist and said, 'Okay, I have to take such and such and such and such, is that okay with what I am taking now?' (P18) Many older adults relied on family caregivers to help with medication management: 'I rely completely on my wife... [She] puts out all my prescriptions on a weekly basis.' (P04) In summary, older adults living with MCC tried to stay as healthy as they could by enacting healthy lifestyles, taking prescribed medications and maintaining social and community connections.

\section{Depending on family caregivers for help with just about everything}

These older adults, who had on average 7.4 chronic conditions, were heavily reliant on family caregivers (primarily spouses and children) for help with many areas of their daily lives including daily living activities and emotional and social support. Participants reported receiving help with a broad range of daily living activities such as personal care (eg, bathing and dressing) as well as instrumental activities (eg, cooking, housekeeping, driving, scheduling and attending appointments, and managing medications). One participant gave this example that clearly illustrates her almost total reliance on her spouse in order to continue living at home:

He [husband] sometimes helps me get dressed on a 'stiff day.' He drives me for most of my errands; he does all the carrying; he does the laundry. Sometimes he'll do the dishes. He helps quite a bit with the housework and with the grandchildren. (P01)

Given the number of medical appointments participants had, not only with their family physicians but also specialists, they relied heavily on others for transportation. This was particularly important when the older adult did not drive, or in situations where their license had been revoked (eg, after a stroke, or when living with dementia): "We have to rely on our two sons or somebody to take us anyplace we want to go. We don't drive.' (P04)

Participants also described how they depended on family and friends for emotional and social support in living with the complexity of MCC. Participants spoke of the strain and stress of living with conditions that worsened with time and that placed them at increased risk of no longer being able to live at home. An older man with dementia spoke of the support he received from his wife as a form of comfort: 'The very fact that we're always together, we do things together, that's the comfort and the emotional support.' (P04) Participants spoke of the value of having someone to talk to about their experiences with MCC so they would not feel alone and isolated in their journey: 'My brother has been through all this. We sit and we talk on the phone every week... and he's always, 'You got to do this; you got to do that, because that's part of what you have to do to make this work." (P14) Participants also described how family helped to motivate them and encourage them to remain independent: 'She [spouse] keeps me motivated in a lot of things. We both try to push each other to independence 
and do what we want to do.' (P14) In summary, participants living with MCC were heavily dependent on their family caregivers for many kinds of support, without which they would be unable to continue living in the community.

\section{Paying the high costs of living with MCC}

Older adults talked extensively about the financial costs of living with MCC. They explained that paying for these costs, whether it was transportation, medications or lost work income, forced them to make choices that negatively impacted their lives and health. Many participants spoke of the high costs of transportation and parking related to their many medical appointments. Some participants spoke of having to pay privately for personal support workers, homemakers or others to assist them with daily living activities so that they could continue to live in their own homes: 'the support persons or unfunded OT/PT [occupational or physical] therapy that my family physician believes would be helpful.' (P10) Participants also described having to pay high costs for special medications that then stretched their ability to pay: 'She [doctor] put me on medication for Alzheimer's. But every month I have to pay for it myself, sixty-nine dollars, so that's something else I want to ask her: Is there another medication that is covered [by the health insurance plan]?' (P21)

Older adults spoke about how they had to carefully manage their finances given the costs associated with living with MCC. Two older adults living with MCC were still working. They reported a struggle in balancing employment and trying to best manage their MCC. One participant explained the tensions experienced in needing to work but having an employer limit time away from work for treatment of MCC: 'clearly, being employed, as far as my employer was concerned, got in the way of me being able to have the treatment options that I needed; painful, but true.' (P10) One man spoke about the impact of losing contract work due to a recent stroke and the concessions to family lifestyle that were made: 'We both got to be going all the time and that is the problem so we back off a little bit because you do not have the money. If you are $\$ 40000$ less this year, that is $\$ 40000$ less things you are going to do.' (P14) Some participants depended on family for help with managing finances and for financial assistance when they could not make ends meet. An older man with dementia described: 'One son kind of manages the money and lends me money when I need it and then I pay him back when I get my government [pension].' (P08) Overall, participants paid many out-of-pocket expenses related to their MCC and these costs created financial hardships for themselves and their families. Further, employed participants experienced workplace restrictions that actually constrained their ability to receive needed treatment, representing a high cost to pay for living with MCC.

\section{Making healthcare decisions by proxy}

Older adults living with MCC made many healthcare decisions by proxy, that is, the decisions were made by either their HCPs or by the distress associated with symptoms they experienced. Many participants indicated that they were not active participants with their healthcare team in making decisions related to their healthcare. Instead, they accepted decisions made by HCPs, in particular, physicians. '[I] try to do what the doctor tells me to do.' (P02) Their comments often reflected their own lack of understanding of their conditions and their perceptions of HCPs as the experts in care; 'I don't question things. They [nurses, doctors, assistants] tell me to do something and I do. It's okay because I don't know much about it.' (P17) Many older adults explained that it was their choice to leave personal care decisions in the hands of HCPs: 'I pretty well leave it up to the doctors. ...I'm always told about it but the decision, I don't think is mine.' (P20) Participants indicated that medication-related decisions were also made by a physician, or between HCPs, without involving the older adult: Oh, the doctor makes those - any decisions with medications.' (P05) Older adults indicated that they accepted and followed doctor orders: 'There was no conversation about it. She [doctor] wrote a prescription for that special pill and I accept what she says.' (P21)

However, a small number of participants explained that being part of the decision-making process was important to them, "Other people might be very happy and feel very supported if they leave it in their doctor's hands... but that doesn't work for me.' (P10) These participants described a more patient-centred approach of informed decision-making with HCPs, 'We [older adult and family doctor] decided together.' (P01) In some situations, collaborative decision-making occurred around medications, 'I discussed the insulin with [Diabetes RN] and with the dietitian, with [the doctor]. My thinking is you guys are the experts so if you think I should try it, I'll try it.' (P09)

Participants also made healthcare decisions based on how distressing their symptoms of MCC were: 'The acute symptoms are managed first' (P10); 'Whatever's the most painful...the most persistent.' (P13) In only a few cases, did participants describe a decision-making process that carefully considered the broader and longer term effects of how they set priorities of care: 'The diabetes would get the first [priority] because it affects everything else...your eyes and your organs and your kidneys.' (P09) Overall, participants made healthcare decisions by proxy, either following HCP orders or according to the distress level of symptoms associated with MCC. Among these participants, there was minimal evidence of taking a lead or co-role in healthcare decision-making based on a sound understanding of the complexity of their chronic conditions.

\section{Receiving healthcare services that do not address the complex needs of persons living with MCC}

These older adults, living with an average of 7.4 chronic conditions, received healthcare services not only from their family physician, but also from 2 to 7 physician 
specialists as well as other HCPs (eg, nurses, chiropractors, personal support workers). They reported that the healthcare services received did not address their complex needs. Services were often experienced as piecemeal, focusing on single physical conditions, rather than on the interaction of all their chronic conditions and seldom attending to their holistic psychological and social needs as a person living with MCC:

You wind up going to all these extra people for each condition which I have found difficult. You don't know them well. You don't see them that often. You kind of feel that you are whatever you have so, let's say if you go to a Rheumatologist, you only want to talk about your arthritis, you don't want to talk about anything else. But it's frustrating...everything's interconnected. (P01)

Participants accepted the reality of this single-condition approach by limiting information-sharing with HCPs, 'When I go to a particular one (specialist) for, say the kidney specialist, they don't ask as to other problems. They're there to take care of that particular one that I'm in there for, so I don't bring it up either' (P19) and 'When I see the Rheumatologist, I don't mention the diabetes... it's like there's little bits of me.' (P13) Participants were subtly coerced to comply with a healthcare approach that denied the complex interweave of their chronic conditions, potentially contributing to further deterioration of their health. One participant eloquently described the impersonal and mechanistic experience of care that made her feel like a 'bad patient' if she hindered the flow of healthcare work: 'I'm not getting the kind of support that I think is appropriate...it's as though I have no emotional needs...I'm sort of marched through like a robot...I'm a bad patient if I hold up the line.' (P10)

When asked, most older adults expressed uncertainty about how their care was coordinated among multiple HCPs. As one participant declared, 'I really don't know and I wonder [how care is coordinated].' (P01) Participants assumed that service providers communicated with each other about their complex care needs, but few could describe how this occurred: 'I guess they [doctors] talk. I don't know.' (P11) Some participants identified their family physician as their care coordinator, the person linking them to other services: 'My family physician is the coordinator of access to all the specialists and all of the allied health professionals so referrals for physio and OT would come through her as well...She and I being the major coordinators of everything and it isn't going to get simpler as I get older.' (P10)

Four participants living with MCC took on the role of coordinating their own care, as the healthcare system did not assume this responsibility. They indicated that they coordinate care by: 'calling and making appointments' (P03), arranging schedules of appointments: 'if that doesn't work, given all the other coordinating that's going on, I just phone the specialist's office and reschedule.' (P10) The responsibility for care coordination was experienced as burdensome and frustrating for participants who already had many demands on their limited time and energy:

I think the frustration... is that so much of what I'm expected to do involves me being my own care coordinator so I'm the one who phones and arranges services and arranges payments...if I could just be the cooperative, participative patient instead of the schedule coordinator, data assembly individual, it would be really helpful. (P10)

Participants also spoke of the lack of communication about their MCC between HCPs and their own need to fill that gap in the system: 'It's sort of up to me to advise [doctors] about health conditions.' (P18) In summary, older adults living with MCC experienced the healthcare system as unable to address their complex physical and emotional needs, and either fell in line with the expectations of the system or took on missing roles (eg, care coordination) themselves.

\section{DISCUSSION}

This study provides a broad description of the experiences of a particularly vulnerable group of older adults living in the community with, on average, 7.4 chronic conditions. Findings indicate that older adults use a range of strategies to stay healthy despite living with MCC that are worsening over time. Further, there is a complex interplay between living with MCC and the social determinants of health such as income and transportation. These older adults rely extensively on family caregivers for support with managing the demands of MCC in order to continue living in the community. Finally, older adults with MCC are seldom actively involved in planning their care with their HCPs, and experience a lack of comprehensive and coordinated care.

Older adults with MCC were recruited from multiple community settings, enhancing credibility. ${ }^{20}$ The sample varied by age, gender, education, income and number and type of chronic conditions, and, unlike other studies, included older adults with memory impairment. Study credibility was enhanced through a rigorous analysis process that involved regular meetings of three investigators and key strategies to enhance rigour. The use of a qualitative ID design facilitated the examination of the complexity and variation of the research phenomenon. ${ }^{30}$

There were a number of study limitations that should be considered. First, all participants were Caucasian and English-speaking, limiting transferability of study findings to other cultural groups. While participants were recruited through a number of sources, most $(71 \%)$ were recruited from two primary care practices. As with all qualitative research, any claims to generalisability are tenuous, and transferability is best suited to similar settings and samples. We did not collect information on participants' responses to the mailed summary of findings, which may have provided a valuable insight into 
their perceptions of the results. Finally, while we used reflexivity to remain aware of our own analytic focus and past experience with the topic, it is possible that these may have subtly shaped study findings.

Study findings make a new contribution to our understanding of the heavy financial costs older adults pay when living with MCC, costs related to transportation, medication and lost income, for example. For employed older adults, findings suggest that workplace demands may even constrain receipt of valued treatment for MCC. A recent integrative review found that current research on multimorbidity provides limited consideration of the role that social determinants of health, such as finances, play in the experiences of persons with $\mathrm{MCC}^{31}$ and this study helps to fill that gap in understanding. Given that onset of multimorbidity has been shown to occur 10-15 years earlier for people living in the most socioeconomically deprived areas, ${ }^{32}$ these findings have implications for health and social supports for community-living older adults with MCC as well as policies to support adequate incomes and enhanced transportation.

Study findings also contribute to our understanding of how heavily reliant these older adults are on their family caregivers, both spouses and children, for support with activities of daily living as well as emotional and social support. This very broad type of caregiver support makes it possible for older adults with multiple and complex chronic conditions to remain in the community, rather than have to move to long-term care facilities. Given the key role that family caregivers play, it is imperative that HCPs engage them in discussions about how best to support older adults with MCC and how to support caregivers to avoid negative outcomes such as caregiver strain, burden and depression.

Study findings make a new contribution to our understanding of how older adults living with MCC make healthcare decisions, that is, largely by proxy, through either following physician recommendations or by attending to the most distressing symptoms of MCC. These approaches preclude active participation of older adults in healthcare decisions that take into consideration the complex interaction of MCC and the longer term impacts of decisions on their health. While recent research indicates that older adults prefer to actively participate in making healthcare decisions, there is evidence that individuals with four or more conditions and those with multiple condition clusters are less likely to prefer active decision-making. ${ }^{33}$ Finally, our study found that older adults received care from family physicians and multiple physician specialists, resulting in experiences of fragmented care. This is congruent with recent evidence that similarly found experiences of fragmented care among this population. ${ }^{173435}$

Study findings suggest that HCPs need to understand and respond to the complexities that older adults experience in living with MCC in order to better support them in their care. This includes assessing how older persons as well as their families are dealing with MCC and discussing issues such as social support, finances and transportation as key factors that influence their ability to manage MCC. There is a need for HCPs to provide enhanced support and guidance to older adults with MCC and their caregivers in taking an active role in their care and care decisions. ${ }^{17}$ This requires strategies to: (a) improve communication and information-sharing, ${ }^{817}$ (b) engage older adults and family caregivers in care-planning discussions ${ }^{17}$ and (c) facilitate care-planning and the integration of services between HCPs. ${ }^{35}$ Tailored written information, and a care plan that incorporates patients' priorities and assists them to locate resources, have helped to support self-management of MCC. ${ }^{10}$ Further, HCPs are challenged to use a more holistic approach to caring for persons with MCC, beyond a single condition focus. This approach would be supported by more comprehensive models of care such as Guided Care, the Program of All-inclusive Care for the Elderly and the IMPACT clinic. ${ }^{36}{ }^{37}$ These models of care involve not just adding more services (eg, more interprofessional team members involved in care) but also fundamentally restructuring the way care is provided. The IMPACT clinic, for example, involves 1.5-hour to 2-hour patient appointments where a diverse range of medical, functional and psychosocial issues are addressed by an extended primary care team including family physicians, a community nurse, a pharmacist, a physiotherapist and occupational therapist, a dietitian and a community social worker.

There are a number of implications for future research in this area. There is a need to better understand the experiences of culturally diverse community-living older adults with MCC, and to explore gender differences in more depth. ${ }^{12}$ An understanding of the similarities and differences of MCC experiences can help HCPs to tailor approaches to the needs of different groups. Further research is also needed to understand the complex interweave of the social determinants of health (eg, income, social support) and the management of MCC. Conceptual models of the role of complexity in the care of persons with MCC may help provide guidance to future research in the area. ${ }^{38}$ Such models demonstrate the scope and breadth of factors that influence the complexity of care of persons with MCC such as personal characteristics, social support, contextual factors (eg, economic), health system and community resources.

Acknowledgements The authors would like to thank all of the study participants and community partners for their contributions to this research. The authors also thank Jennifer Plenderleith, Research Coordinator, for her contributions to the study.

Contributors JP and MM-R obtained funding for the project. JP and KDF conceived of the project, wrote the protocol and obtained ethical approval. JP supervised the overall project. MC, JP and TC analyzed the data. MC wrote the first draft of the manuscript. JP, MC, KDF, CM, SK, MM-R, SD, LGB, and TC reviewed and approved the final manuscript. All authors meet ICMJE criteria for authorship.

Funding This study is part of a program of research (Aging, Community and Health Research Unit, School of Nursing, McMaster University), supported by the Canadian Institutes of Health Research Signature Initiative in Community-Based Primary Healthcare (http://www.cihr-ircs.gc.ca/e/43626.html) (Funding Reference Number: TTF 128261) and the Ontario Ministry of Health and Long-Term Care Health System Research Fund Program (Grant \#06669). MM-R was also supported through the 
Canada Research Chairs Program, as the Chair in Aging, Chronic Disease and Health Promotion Interventions.

Competing interests None declared.

Patient consent for publication Not required.

Ethics approval The study was approved by the Hamilton Integrated Research Ethics Board (\#13-411) in Hamilton, Ontario, Canada.

Provenance and peer review Not commissioned; externally peer reviewed.

Data sharing statement The data for this research consist of in-person interview transcripts. Raw data cannot be publicly released due to the risk of compromising participant confidentiality.

Open access This is an open access article distributed in accordance with the Creative Commons Attribution Non Commercial (CC BY-NC 4.0) license, which permits others to distribute, remix, adapt, build upon this work non-commercially, and license their derivative works on different terms, provided the original work is properly cited, appropriate credit is given, any changes made indicated, and the use is non-commercial. See: http://creativecommons.org/licenses/by-nc/4.0/.

\section{REFERENCES}

1. Bayliss EA, Bonds DE, Boyd CM, et al. Understanding the context of health for persons with multiple chronic conditions: moving from what is the matter to what matters. Ann Fam Med 2014;12:260-9.

2. Boyd CM, Fortin M. Future of multimorbidity research: How should understanding of multimorbidity inform health system design? Public Health Rev 2010;32:451-74.

3. Roberts KC, Rao DP, Bennett TL, et al. Prevalence and patterns of chronic disease multimorbidity and associated determinants in Canada. Health Promot Chronic Dis Prev Can 2015;35:87-94.

4. World Health Organisation. Multimorbidity. 2016 http://apps.who.int/ iris/bitstream/10665/252275/1/9789241511650-eng.pdf (Accessed 28 Dec 2016).

5. Marengoni A, Angleman S, Melis R, et al. Aging with multimorbidity: a systematic review of the literature. Ageing Res Rev 2011;10:430-9.

6. Melis R, Marengoni A, Angleman S, et al. Incidence and predictors of multimorbidity in the elderly: a population-based longitudinal study. PLoS One 2014;9:e103120.

7. Canadian Institute for Health Information (CIHI). Seniors and the health care system: what is the impact of multiple chronic conditions? $2011 \mathrm{https}$ ://secure.cihi.ca/free_products/air-chronic disease_aib_en.pdf (Accessed 28 Dec 2016).

8. Bratzke LC, Muehrer RJ, Kehl KA, et al. Self-management priority setting and decision-making in adults with multimorbidity: a narrative review of literature. Int J Nurs Stud 2015;52:744-55.

9. Coventry PA, Small N, Panagioti M, et al. Living with complexity; marshalling resources: a systematic review and qualitative metasynthesis of lived experience of mental and physical multimorbidity. BMC Fam Pract 2015;16:171.

10. Liddy C, Blazkho V, Mill K. Challenges of self-management when living with multiple chronic conditions: systematic review of the qualitative literature. Can Fam Physician 2014;60:1123-33.

11. Eckerblad J, Theander K, Ekdahl A, et al. To adjust and endure: a qualitative study of symptom burden in older people with multimorbidity. Appl Nurs Res 2015;28:322-7.

12. Clarke LH, Bennett $E$. 'You learn to live with all the things that are wrong with you': gender and the experience of multiple chronic conditions in later life. Ageing Soc 2013;33:342-60.

13. Roberto KA, Gigliotti CM, Husser EK. Older women's experiences with multiple health conditions: daily challenges and care practices. Health Care Women Int 2005;26:672-92.

14. Löffler C, Kaduszkiewicz H, Stolzenbach CO, et al. Coping with multimorbidity in old age--a qualitative study. BMC Fam Pract 2012;13:45

15. Fried TR, McGraw S, Agostini JV, et al. Views of older persons with multiple morbidities on competing outcomes and clinical decisionmaking. J Am Geriatr Soc 2008;56:1839-44.
16. Bayliss EA, Edwards AE, Steiner JF, et al. Processes of care desired by elderly patients with multimorbidities. Fam Pract 2008;25:287-93.

17. Gill A, Kuluski K, Jaakkimainen L, et al. "Where do we go from here?" Health system frustrations expressed by patients with multimorbidity, their caregivers and family physicians. Healthc Policy 2014;9:73-89.

18. Kuluski K, Gill A, Naganathan G, et al. A qualitative descriptive study on the alignment of care goals between older persons with multimorbidities, their family physicians and informal caregivers. BMC Fam Pract 2013;14:133.

19. Ploeg J, Matthew-Maich N, Fraser K, et al. Managing multiple chronic conditions in the community: a Canadian qualitative study of the experiences of older adults, family caregivers and healthcare providers. BMC Geriatr 2017;17:40.

20. Thorne S. Interpretive description: qualitative research for applied practice. 2nd edn. New York, NY: Routledge, 2016.

21. Thorne S, Kirkham SR, O'Flynn-Magee K. The analytic challenge in interpretive description. Int J Qual Methods 2004;3:1-11.

22. Statistics Canada. Mortality, summary list of causes, 2009. 2012 http://www5.statcan.gc.ca/olc-cel/olc.action?Objld=84F0209X\& ObjType=2\&lang=en\&limit=0 (Accessed 28 Dec 2016)

23. World Health Organisation. Cardiovascular diseases (CVDs) Fact sheet. 2017 http://www.who.int/mediacentre/factsheets/fs317/en/ (Accessed 28 Dec 2016).

24. Braun V, Clarke V. Using thematic analysis in psychology. Qual Res Psychol 2006;3:77-101.

25. Thorne S, Kirkham SR, MacDonald-Emes J. Interpretive description: a noncategorical qualitative alternative for developing nursing knowledge. Res Nurs Health 1997;20:169-77.

26. Lincoln YS, Guba EG, Pilotta JJ. Naturalistic inquiry. . Newbury Park, CA: Sage, 1985:9. 438-9.

27. Patton M. Qualitative research and evaluation methods: integrating theory and practice. 4th ed. Thousand Oaks, CA: Sage, 2015.

28. Canadian Institutes of Health Research, Natural Sciences and Engineering Research Council of Canada, Social Sciences and Humanities Research Council of Canada. Tri-Council Policy Statement: Ethical Conduct for Research Involving Humans. Canadian Institutes of Health Research, Natural Sciences and Engineering Research Council of Canada, Social Sciences and Humanities Research Council of Canada. 2014 http://www. pre.ethics.gc.ca/pdf/eng/tcps2-2014/TCPS_2_FINAL_Web.pdf (Accessed 20 Mar 2018)

29. Cohen-Mansfield J. Consent and refusal in dementia research: conceptual and practical considerations. Alzheimer Dis Assoc Disord 2003;17(Suppl. 1):S17-S25.

30. Hunt MR. Strengths and challenges in the use of interpretive description: reflections arising from a study of the moral experience of health professionals in humanitarian work. Qual Health Res 2009;19:1284-92.

31. Northwood M, Ploeg J, Markle-Reid M, et al. Integrative review of the social determinants of health in older adults with multimorbidity. $J$ Adv Nurs 2018;74:45-60.

32. Barnett K, Mercer SW, Norbury M, et al. Epidemiology of multimorbidity and implications for health care, research, and medical education: a cross-sectional study. Lancet 2012;380:37-43.

33. Chi WC, Wolff J, Greer R, et al. Multimorbidity and decision-making preferences among older adults. Ann Fam Med 2017;15:546-51.

34. Morris RL, Sanders C, Kennedy AP, et al. Shifting priorities in multimorbidity: a longitudinal qualitative study of patient's prioritization of multiple conditions. Chronic IIIn 2011;7:147-61.

35. Newbould J, Burt J, Bower P, et al. Experiences of care planning in England: interviews with patients with long term conditions. BMC Fam Pract 2012;13:71.

36. Boult $\mathrm{C}$, Wieland GD. Comprehensive primary care for older patients with multiple chronic conditions: "Nobody rushes you through". JAMA 2010;304:1936-43.

37. Tracy CS, Bell SH, Nickell LA, et al. The IMPACT clinic: innovative model of interprofessional primary care for elderly patients with complex health care needs. Can Fam Physician 2013;59:e148-55.

38. Grembowski D, Schaefer J, Johnson KE, et al. A conceptual model of the role of complexity in the care of patients with multiple chronic conditions. Med Care 2014;52 Suppl 3:S7-S14. 